\title{
Continuous Aspiration Mechanical Thrombectomy for Treatment of Thrombosed Hemodialysis Reliable Outflow Grafts
}

\begin{abstract}
Aims: To determine the safety and feasibility of continuous aspiration mechanical thrombectomy (CAT) for restoring patency to thrombosed hemodialysis reliable outflow (HeRO) arteriovenous grafts. Subjects and Methods: Between December 2016 and August 2017, eleven consecutive patients (average age 63, range 39-80 years) with thrombosed HeRO grafts underwent percutaneous thrombectomy procedures $(n=21)$ using the Penumbra Indigo ${ }^{\circledR}$ CAT 8 or CAT D (Alameda, CA, USA) thrombectomy catheter as the primary device to clear the venous outflow tract before removing the arterial plug with a compliant balloon. A total of 21 hemodialysis declot procedures using CAT were documented and analyzed. Average procedure length and fluoroscopy time, length of thrombus cleared, blood loss, complications, and time between thrombectomy procedures were recorded and compared to the same patient's previous three thrombectomy procedures. Results: All procedures were technically successful $(100 \%)$ at restoring graft patency; however, reocclusion within 5 days occurred in four $(19.0 \%)$ cases. Three $(14.3 \%)$ interventions required additional balloon maceration or sweep to clear the venous outflow following thrombectomy. Average thrombus length treated by suction thrombectomy measured $23.15 \mathrm{~cm}$ (range $2.2-65 \mathrm{~cm}$ ). Average blood loss was $162.6 \mathrm{~mL}$ (range 50-250 mL). No procedure-related complications were recorded. The average procedure length and fluoroscopy time using suction thrombectomy was 74.7 and $14.2 \mathrm{~min}$, respectively, compared with 82.0 and $14.0 \mathrm{~min}$, respectively, in the previous thrombectomy procedures using standard methods $(P>0.05)$. Seventeen $(81 \%)$ HeRO grafts treated by CAT presented with rethrombosis at a mean of 42.47 days (range 1-208 days, median 22 days, standard deviation [SD] 28.2 days) since CAT procedure compared to patients treated by conventional methods who presented for rethrombosis at a mean of 55.33 days (range 1-321 days, median 34 days, SD 43.1 days) since standard thrombectomy - no statically significant difference $(P>0.05)$. Conclusion: CAT is a safe and feasible method for removing thrombus and restoring patency to thrombosed HeRO grafts. Further studies are required to elucidate the advantages of CAT over standard thrombectomy techniques.
\end{abstract}

Keywords: Endovascular, hemodialysis, suction, thrombectomy

\section{Introduction}

Hemodialysis reliable outflow (HeRO) grafts (Merit Medical; South Jordan, Utah, USA) are devices often used in patients with failed alternative methods of achieving arteriovenous (AV) access such as $\mathrm{AV}$ fistulas or conventional polytetrafluoroethylene (PTFE) AV grafts. HeRO grafts consist of two components; ${ }^{[1]}$ expanded PTFE in the upper arm anastamosed to the brachial artery and connected to a braided nitinol outflow component with its tip in the right atrium. ${ }^{[2,3]}$ This lengthy direct shunt between the artery and right atrium poses potential risk for greater thrombus burden

This is an open access journal, and articles are distributed under the terms of the Creative Commons Attribution-NonCommercial-ShareAlike 4.0 License, which allows others to remix, tweak, and build upon the work non-commercially, as long as appropriate credit is given and the new creations are licensed under the identical terms.

For reprints contact: reprints@medknow.com when compared to conventional PTFE AV grafts. ${ }^{[4]}$ This thrombus burden ultimately further increases the risk of subclinical pulmonary emboli during thrombectomy procedures which can result in chronic pulmonary hypertension. ${ }^{[5-9]}$ This potential consequence of HeRO graft thrombectomy emphasizes the need to minimize the extent of subclinical pulmonary embolism (PE), particularly in patients with large clot volume.

Percutaneous continuous aspiration thrombectomy interventions for mechanical thrombectomy are well documented in the literature and have been shown to be safe and effective as both primary and adjunctive endovascular therapies for treating acute thromboembolic disease. In

\footnotetext{
How to cite this article: Ahmed O, Hadied MO, Madassery S, Ozen M, Tran P, Lizardo AE, et al. Continuous aspiration mechanical thrombectomy for treatment of thrombosed hemodialysis reliable outflow grafts. Arab J Intervent Radiol 2020;4:21-6.
}

\section{Osman Ahmed, Mohamad Omar Hadied ${ }^{1}$, Sreekumar Madassery, Merve Ozen, Patrick Tran, Adolfo E. Lizardo², Jordan C. Tasse, Ulku Cenk Turba, Bulent Arslan}

Department of Radiology, Section of Interventional Radiology, Rush University Medical Center, Chicago, IL, ${ }^{\prime}$ Department of Radiology, Wayne State University School of Medicine, Detroit, MI, USA, ${ }^{2}$ Department of Radiology, South Medical Clinic and Hospital, National Institute of Cancer, Mexico City, Mexico

Received: 27-05-2019 Revised: 30-05-2019 Accepted: 08-06-2019 Published Online: 25-07-2019

Address for correspondence: Dr. Osman Ahmed,

Rush University Medical Center, 5841 S. Maryland Ave., Chicago 60637, IL, USA.

E-mail: osman1423@gmail.com

Access this article online

Website: www.arabjir.com

DOI: 10.4103/AJIR.AJIR_9_19

Quick Response Code: 
the last 5 years, numerous studies have described the use of continuous aspiration mechanical thrombectomy (CAT) for both arterial and venous applications. Specifically, the role for CAT has been identified as a treatment for PE, lower extremity arterial occlusions, carotid occlusions, iliocaval thromboses, right atrial thromboses, mesenteric thromboses, as well as aspiration of vegetations on implanted cardiac leads or valves. ${ }^{[1,2,10-14]}$ Given its potential to evacuate thrombus and prevent recurrent subclinical pulmonary emboli, CAT may represent a method to treat thrombosed dialysis access shunts. Currently, however, the role of CAT for restoring patency in dialysis access has not been clearly defined. The purpose of this study was therefore to investigate the safety and feasibility of using CAT to restore patency to thrombosed HeRO access grafts.

\section{Subjects and Methods}

\section{Patients}

A retrospective study, approved by the Rush University Medical Center Institutional Review Board, of all consecutive patients undergoing percutaneous thrombectomy for thrombosed HeRO grafts between December 2016 and September 2017 was conducted at a tertiary care academic hospital. Informed written consent was obtained from all patients for this study. At our institution, the standard approach to diagnose the thrombosed graft is made clinically by physical examination indicating a lack of thrill. As per institutional preference, mechanical thrombectomy devices are used routinely to treat clotted HeRO grafts. Among these cases, patients who had undergone thrombectomy using the Penumbra ${ }^{\circledR}$ (Alameda, CA, USA) Indigo CAT system were identified. Inclusion criteria included patients with HeRO grafts who had undergone at least three prior thrombectomies using standard mechanical thrombectomy techniques (to allow for comparison). Demographic and procedural variables were recorded from the patients' electronic medical charts and picture archiving and communication system database [Table 1]. Complications were documented according to Society of Interventional Radiology quality improvement guidelines for percutaneous image-guided management of the thrombosed or dysfunctional dialysis circuit. ${ }^{[15]}$

In the time period studied, a total of 183 percutaneous pharmacomechanical thrombectomies for thrombosed dialysis access shunts were performed. Among these, percutaneous thrombectomy using CAT was performed 21 times in 11 consecutive patients with HeRO grafts. Patients with HeRO grafts identified to be clotted within 1 week of presentation were treated using the CAT-D or CAT-8 Indigo $^{\circledR}$ aspiration device (Penumbra ${ }^{\circledR}$, Alameda, CA, USA). Standard institutional protocol for performing percutaneous thrombectomy on thrombosed dialysis $\mathrm{AV}$ shunts included correction of serum potassium to a level $<6 \mathrm{mmol} / \mathrm{L}$ and an international normalized

\begin{tabular}{lc}
\hline \multicolumn{2}{c}{ Table 1: Patient demographics } \\
\hline Variables & $\boldsymbol{n}(\mathbf{\%})$ or (range) \\
\hline Male & $6(54.54)$ \\
Female & $5(45.45)$ \\
Age, years & $63(39-80)$ \\
Mean length of ESRD & 9.65 \\
Comorbidity & \\
Hypertension & $11(100)$ \\
Hyperlipidemia & $5(45.45)$ \\
Diabetes mellitus & $4(36.36)$ \\
Antiplatelet therapy & \\
Aspirin & $9(81.81)$ \\
Clopidogrel (plavix) & $6(54.54)$ \\
\hline
\end{tabular}

ESRD: End-stage renal disease

ratio $<3$. All CAT procedures were performed by one of three board-certified interventional radiologists with at least 3 years of experience. The follow-up duration was 6 months for all patients.

Continuous aspiration mechanical thrombectomy devices

The CAT system consists of four components: a catheter, separator wire, reinforced tubing, and an aspiration pump. This study utilized the CAT-D $(50 \mathrm{~cm})$ and CAT- $8(80 \mathrm{~cm})$ catheters which are both eight French in size. The reinforced tubing is a tube which connects the catheters to the portable pump to provide continuous aspiration. The separator is a wire that aids in agitation and debulking of thrombus to allow for more efficient aspiration.

\section{Procedure technique}

Thrombosed grafts were initially accessed toward the venous limb near the $\mathrm{AV}$ anastomosis using a micropuncture set $\left(\right.$ Cook $^{\circledR}$, Bloomington, IN, USA). An eight French sheath (Prelude ${ }^{\circledR}$; Merit Medical ${ }^{\circledR}$, South Jordan, UT) was subsequently placed over a stiff 0.035 " guidewire. A five French angled catheter and hydrophilic guidewire were advanced into the right atrium, and pull back venography was performed to delineate the extent of thrombus and also to measure its length [Figure 1]. Next, a $65 \mathrm{~cm}$ total length infusion catheter (20 $\mathrm{cm}$ infusion length) was advanced to span across the length of thrombus. Four milligram of tissue plasminogen activator (TPA) (Activase ${ }^{\circledR}$; Genentech, San Francisco, CA) diluted into $20 \mathrm{ml}$ of heparinized saline and contrast was pulse sprayed into the infusion catheter in $1 \mathrm{ml}$ aliquots and allowed to dwell for $10 \mathrm{~min}$.

Following TPA dwell, the CAT-D $(n=11)$ or CAT-8 $(n=10)$ device was placed and used to perform CAT throughout the graft [Figure 2]. Up to five passes of the device were performed based on operator preference and predetermined total blood loss not exceeding $250 \mathrm{ml}$ during device activation. No prophylactic blood transfusions were performed.

Subsequently, a second access was obtained directed toward the arterial limb of the graft followed by placement 


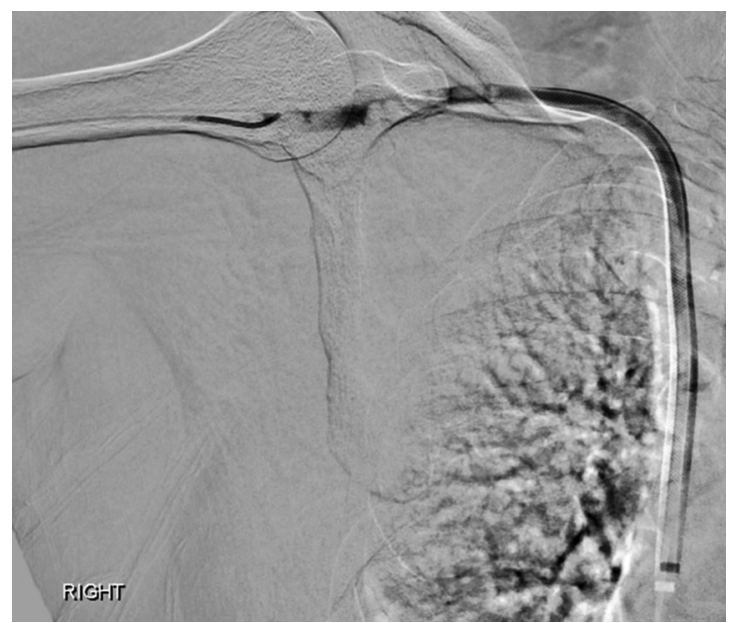

Figure 1: Pull-back venogram delineating central extent of thrombus burden at the level of the venous outflow connector

of a six French sheath. An angled catheter was advanced beyond the anastomosis into the native brachial artery, and arteriography was performed from this position to assess the clot burden within the graft. Next, the arterial plug of the graft was pulled into the graft using a compliant five French Fogarty balloon over a 0.035 " guidewire. Attention was paid not to displace any thrombus into the artery. Repeat arteriography was performed to confirm restored patency of the graft [Figure 3]. Any underlying stenosis treated by balloon angioplasty was recorded. Adjunctive techniques to clear thrombus such as balloon maceration or sweep were also recorded. Completion angiography was performed to confirm no residual thrombus or stenosis throughout the graft [Figure 3]. A minimum of 5000 IU intravenous heparin was administered during the procedure for systemic anticoagulation. All devices and catheters were removed at the conclusion of the procedure. Hemostasis was obtained using purse-string suture technique around the eight French access and manual compression of the six French access. Patients were discharged following routine $4 \mathrm{~h}$ postprocedure monitoring.

Conventional methods for HeRO graft thrombectomy involved a similar method of graft access with utilization of a thrombectomy device (Arrow-Treotola ${ }^{\mathrm{TM}}$; Teleflex Medical: Wayne, PA and Cleaner ${ }^{\circledR}$; Argon Medical: Frisco, TX) and TPA to clear the venous outflow before pulling the arterial plug of the graft. Graft access was maintained with a 7 French and 6 French sheath for the venous and arterial limbs, respectively.

\section{Outcome definitions}

Technical success (with or without adjunctive methods) was defined as restored graft patency at the conclusion of the procedure with palpable thrill. Thrombus length cleared by CAT was also calculated using a $5 \mathrm{~mm}$ assumption for graft diameter of the venous outflow component and $6 \mathrm{~mm}$ for the arterial component.

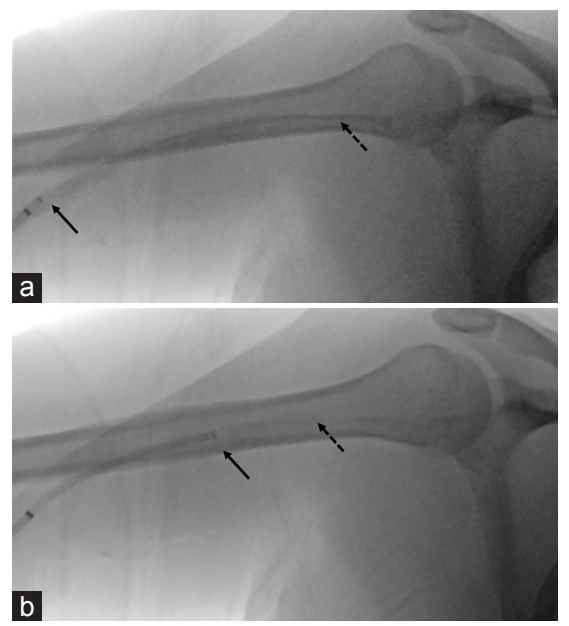

Figure 2: Spot radiograph taken during continuous aspiration mechanical thrombectomy using the continuous aspiration mechanical thrombectomy- $D$ thrombectomy device - before (a) and after (b) device (arrow) was turned on and advanced. Note contrast with thrombus cleared on before and after images (dashed arrow)

\section{Statistical analysis}

All statistical analyses were conducted using Microsoft ${ }^{\circledR}$ Excel 2016 (Microsoft $^{\circledR}$, Redmond, WA). The formula used to determine the potential volume of clot removed was the standard volume of a right cylinder $\left(V=\pi r^{2} h\right)$. Paired one-tail distribution $t$-tests were conducted using recorded patient and procedural information from the medical record. Procedure and fluoroscopy times using conventional methods for thrombectomy were compared against the CAT method in the same patients. Time to rethrombosis between prior thrombectomy procedures using conventional techniques were also compared to CAT in the same patients. As all patients in the cohort studied underwent thrombectomy at least 3 times previously using institutional routine methods, the average of these three prior thrombectomies was chosen for the values reported in the "conventional" group. $P<0.05$ was considered as statistically significant.

\section{Results}

All procedures were technically successful $(21 / 21,100 \%)$. No procedure-related complications occurred. Reocclusion within 5 days occurred in four $(19.0 \%)$ cases, with two patients presenting with rethrombosis within $24 \mathrm{~h}$. Three (14.3\%) interventions required additional balloon maceration or sweep as an adjunctive therapy to clear the venous outflow following thrombectomy. The average thrombus length treated measured $23.15 \mathrm{~cm}$. The average $23.15 \mathrm{~cm}$ of thrombus treated represented $6.54 \mathrm{~mL}$ of potential clot volume removed. The average blood loss was $162.6 \mathrm{~mL}$. Seventeen $(81.0 \%)$ HeRO grafts treated by CAT presented with rethrombosis of the graft at a mean of 42.47 days since CAT - procedural details are elucidated in Table 2. Average time to rethrombosis in the same patients using conventional methods was 55.33 days, not statistically significant $(P>0.05)$. 
The average procedure length and fluoroscopy time using CAT was 74.7 and $14.2 \mathrm{~min}$, respectively, compared with 82.0 and $14.0 \mathrm{~min}$, respectively, in the previous thrombectomy procedures using standard methods $(P>0.05)$ - detailed comparison of CAT and standard thrombectomy is summarized in Table 3.

\section{Discussion}

The incidence of patients requiring thrombectomy to salvage thrombosed dialysis access shunts has been increasing over the past decade. ${ }^{[16]} \mathrm{HeRO}$ grafts are particularly prone to thrombosis since they are essentially a long segment graft extending from a target artery to the right atrium without a venous anastomosis. Wallace et al. reviewed HeRO grafts and showed that all successfully placed grafts in the study had thrombosed within 4 months of placement. ${ }^{[17]}$ Another small study with long-term follow-up of 38 HeRO grafts showed primary patency of $38.9 \%$ and a reintervention rate of 2.5 times/year. ${ }^{[18]}$ Ladenheim et al. retrospectively analyzed $40 \mathrm{HeRO}$ grafts and showed a similar primary patency rate of $30 \%$ with an average intervention rate of 2.1 times/year. ${ }^{[19]}$ A pooled review of multiple similar studies including $409 \mathrm{HeRO}$ grafts confirmed similar results, demonstrating $21.9 \%(9.6-37.2)$ primary patency rate and an intervention rate of 1.5-3 times/year. ${ }^{[20]}$

Current methods for performing thrombectomy procedures include push-pull thrombectomy using an occlusion balloon, rotational thrombectomy, rheolytic thrombectomy, and balloon angioplasty for clot maceration. ${ }^{[21]}$ These techniques all depend on clot maceration with the remainder of thrombus in the vessel to result in potential downstream complications. PE associated with the HeRO grafts have been reported up to $2.6 \% .^{[18,22]} \mathrm{PE}$ following HeRO thrombectomy procedures using current methods are rare but have been reported. Gebhard et al. reported a major complication of $\mathrm{PE}$ and death 2 days following HeRO thrombectomy using TPA and the push-pull thrombectomy

\begin{tabular}{lcc}
\hline \multicolumn{3}{c}{ Table 2: Operative data } \\
\hline Mean \pm SD & Median (range) \\
\hline Thrombus length treated, $\mathrm{cm}$ & $23.15 \pm 19.6$ & $20(2.2-65)$ \\
Blood loss, $\mathrm{mL}$ & $6.54 \pm 5.54$ & $5.65(0.62-18.38)$ \\
Fluoroscopy time, min & $162.6 \pm 51.4$ & $150(50-250)$ \\
Procedure time, min & $14.2 \pm 9.10$ & $11.6(3.5-29.7)$ \\
\hline
\end{tabular}

SD: Standard deviation technique. ${ }^{[3]}$ The estimated volume of thrombus released during a typical dialysis thrombectomy was estimated in this study to be $1.6-4.7 \mathrm{~mL}^{[3]}$

There have been multiple studies aimed at assessing the burden of released thromboemboli following repeated thrombectomy for thrombosed hemodialysis accesses. ${ }^{[18,23-26]}$ In addition, the hemodynamic consequences of thrombus embolization (excluding symptomatic PE) have also been investigated. Hsieh et al. reported a prospective study on 52 patients assessing pulmonary arterial pressures (PAP) following repeated thrombectomy procedures and found no correlation with elevated PAP and repeated thrombectomy in the short term. ${ }^{[27]}$ While similar studies have been reported supporting this finding, ${ }^{[9,28,29]}$ the long-term effects of frequent thrombectomy procedures and potential embolic thrombus they produce with regard to chronic pulmonary hypertension and its related cardiovascular compromise remain largely unknown.

The poor patency rates for HeRO grafts reported in literature put affected patients at an increased risk of mechanical thrombectomy-associated complications. Given a larger thrombus burden and increased frequency of reintervention, this patient population may be at greater risk for subclinical PE and associated chronic pulmonary hypertension. The current study investigated the feasibility of continuous aspiration thrombectomy as a method for thrombectomy to salvage thrombosed HeRO grafts. Technical success was found to be $100 \%$ with no major or minor procedural complications recorded. Of

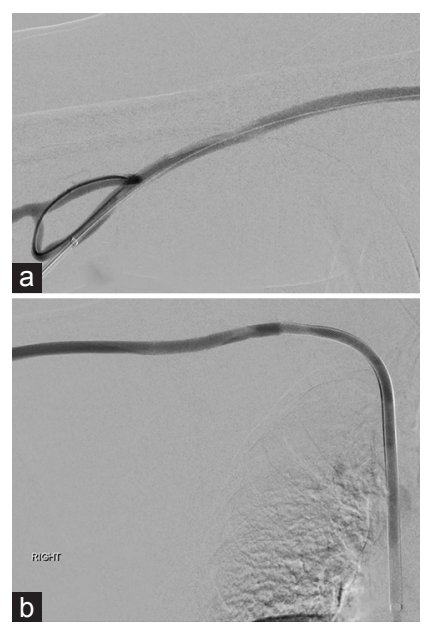

Figure 3: Brachial (a) and central (b) venogram demonstrating restored patency throughout the graft

\begin{tabular}{|c|c|c|c|c|c|}
\hline & \multicolumn{2}{|c|}{ Mean \pm SD } & \multicolumn{2}{|c|}{ Median (range) } & \multirow[t]{2}{*}{$P$} \\
\hline & Non-CAT & CAT & Non-CAT & CAT & \\
\hline Time to repeat thrombectomy, days & $55.3 \pm 43.1$ & $42.5 \pm 58.2$ & $34(1-321)$ & $22(1-208)$ & $>0.05$ \\
\hline Fluoroscopy time, min & $14.0 \pm 6.6$ & $14.2 \pm 9.1$ & $17.4(1.8-38)$ & $11.6(3.5-37)$ & $>0.05$ \\
\hline Procedure time, min & $82.0 \pm 32.2$ & $74.7 \pm 33.6$ & $66(21-238)$ & $67(21-147)$ & $>0.05$ \\
\hline
\end{tabular}

SD: Standard deviation, CAT: Continuous aspiration mechanical thrombectomy 
21 cases included, 17 required follow-up procedures for rethrombosis of the graft; mean patency of dialysis graft was 42.47 days compared to 55.33 days using conventional methods previously on the same patients. Patency in days across techniques was found to not be statistically different $(P>0.05)$. Given the aforementioned established high rates of rethrombosis for HeRO grafts among the end-stage renal disease (ESRD) population, percutaneous CAT for HeRO graft thrombectomy was found to be a safe and effective tool to provide durable patency and restore access for patients with challenging venous access.

Fluoroscopy and procedural times were also analyzed comparing conventional methods for thrombectomy compared to CAT. Slightly lower overall procedure times were measured ( 82.0 vs. $74.7 \mathrm{~min}$ ) using CAT compared to the conventional methods utilized at the authors' institution; however, this result was not statistically significant. A theoretical advantage of the utilized CAT method includes the opportunity to reduce the risk for thrombus embolization centrally. In the current study involving 21 cases, the average length of clot treated was $23.15 \mathrm{~cm}$ and, when converted to a representative volume, the potential volume of clot removed averaged $6.54 \mathrm{~mL}$ $(0.62-18.38 \mathrm{~mL})$. Although not specifically investigated, CAT for percutaneous thrombectomy may theoretically reduce PE risk by allowing for physical removal of the majority of thrombus burden. Removal of thrombus burden may also have implications on the use of CAT for percutaneous thrombectomy of hemodialysis accesses when conventional percutaneous mechanical thrombectomy would otherwise be contraindicated by preexisting pulmonary hypertension or compromised cardiopulmonary function.

This study is limited by its retrospective nature, small sample size, and single institutional experience. Statistical significance of reported results is also limited by the power of a small sample size. Procedural and technical skill differences between operators may exist and could at least partially explain potential differences in fluoroscopy and procedure time. Given that patients with ESRD suffer from anemia of chronic kidney disease, the CAT method was limited by judicious application time to minimize blood loss. The average blood loss was $162.6 \mathrm{~mL}$ (range 50-250 mL), but no blood products were required during or after the procedure. In addition, the proposed benefits of thrombus evacuation are theoretical as the current study did not specifically investigate the benefits of CAT compared to conventional methods with respect to $\mathrm{PE}$ or pulmonary hypertension. Furthermore, the results for clot removal volume represented a theoretical maximum of clot volume removed as the amount of thrombus was not actually weighed or quantified during the procedure. Additional limitations of the study included a high percentage of patients with rethrombosis within 5 days of the procedure. This was partially thought to be due to the learning curve of operators using an 8 French sheath when obtaining hemostasis (not standard at the authors' institution), which required a purse string at the puncture site compared to our routine manual compression. Furthermore, the cost-effectiveness of CAT was not investigated in this study and may contribute to its overall adoption as a feasible device for this patient population. Finally, the investigated CAT technique also utilized TPA and occasional balloon maceration as an adjunctive tool for restoring graft patency. Further exploration of the CAT method without these agents may mitigate the cost associated with using the thrombectomy device.

\section{Conclusion}

In conclusion, percutaneous CAT is a safe and feasible method for salvage of thrombosed HeRO grafts. This method requires further investigation to establish its potential advantage over other conventional techniques, namely in its ability to extract thrombus en bloc and prevent downstream embolization.

Financial support and sponsorship

Nil.

\section{Conflicts of interest}

Osman Ahmed: Speaker, Bard/Penumbra/Argon Medical; Advisory Board Member, BTG/Abbvie. Sreekumar Madassery: Speaker, Abbott; Speaker, Cook. Bulent Arslan: Speaker and advisory board member for Penumbra ${ }^{\circledR}$, Medtronic/Covidien ${ }^{\circledR}$, and speaker for Cook $^{\circledR}$, W.L. Gore ${ }^{\circledR}$, Guerbet $^{\mathbb{}}$, and CR Bard ${ }^{\circledR}$.

\section{References}

1. Young $\mathrm{S}$, Pritzker $\mathrm{M}$, Rosenberg $\mathrm{M}$. Vacuum-assisted thrombectomy of massive pulmonary embolism. J Vasc Interv Radiol 2016;27:1094-6.

2. Baumann F, Sharpe E $3^{\text {rd }}$, Peña C, Samuels S, Benenati JF. Technical results of vacuum-assisted thrombectomy for arterial clot removal in patients with acute limb ischemia. J Vasc Interv Radiol 2016;27:330-5.

3. Gebhard TA, Bryant JA, Adam Grezaffi J, Pabon-Ramos WM, Gage SM, Miller MJ, et al. Percutaneous interventions on the hemodialysis reliable outflow vascular access device. J Vasc Interv Radiol 2013;24:543-9.

4. Vieira M, Ferreira T, Fortes A, Marins P, Ministro A, Ferreira H, et al. Hemodialysis Reliable Outflow (HeRO) Graft device: A lifesaving solution in multiple vascular access failure in haemodialysis patients. Port J Nephrol Hypert 2016;30:217-22.

5. Yigla M, Abassi Z, Reisner SA, Nakhoul F. Pulmonary hypertension in hemodialysis patients: An unrecognized threat. Semin Dial 2006;19:353-7.

6. Havlucu Y, Kursat S, Ekmekci C, Celik P, Serter S, Bayturan O, et al. Pulmonary hypertension in patients with chronic renal failure. Respiration 2007;74:503-10.

7. Centers for Medicare and Medicaid Services, Kinney R 2005 annual report: ESRD clinical performance measures project. Am J Kidney Dis 2006;48:S1-106.

8. Smits HF, Smits JH, Wüst AF, Buskens E, Blankestijn PJ. Percutaneous thrombolysis of thrombosed haemodialysis access grafts: Comparison of three mechanical devices. Nephrol Dial 
Transplant 2002;17:467-73.

9. Petronis JD, Regan F, Briefel G, Simpson PM, Hess JM, Contoreggi CS, et al. Ventilation-perfusion scintigraphic evaluation of pulmonary clot burden after percutaneous thrombolysis of clotted hemodialysis access grafts. Am J Kidney Dis 1999;34:207-11.

10. Rigatelli G, Martire G, Gentile M, Michielan F, Amistà $\mathrm{P}$. Combined use of export catheter and penumbra vacuum thromboaspiration in a challenging case of acute common carotid artery occlusion. Cardiovasc Revasc Med 2016;17:468-9.

11. Smith SJ, Behrens G, Sewall LE, Sichlau MJ. Vacuum-assisted thrombectomy device (AngioVac) in the management of symptomatic iliocaval thrombosis. J Vasc Interv Radiol 2014;25:425-30.

12. Al Badri A, Kliger C, Weiss D, Pirelli L, Wilson S, DeLaney ER, et al. Right atrial vacuum-assisted thrombectomy: Single-center experience. J Invasive Cardiol 2016;28:196-201.

13. Monastiriotis S, Gonzales C, Kokkosis A, Labropoulos N, Bilfinger $\mathrm{T}$, Tassiopoulos AK, et al. The use of angioVac for symptomatic aortic thrombus complicated by mesenteric ischemia. Ann Vasc Surg 2016;32:129.e1-6.

14. George B, Voelkel A, Kotter J, Leventhal A, Gurley J. A novel approach to percutaneous removal of large tricuspid valve vegetations using suction filtration and veno-venous bypass: A single center experience. Catheter Cardiovasc Interv 2017;90:1009-15.

15. Dariushnia SR, Walker TG, Silberzweig JE, Annamalai G, Krishnamurthy V, Mitchell JW, et al. Quality improvement guidelines for percutaneous image-guided management of the thrombosed or dysfunctional dialysis circuit. J Vasc Interv Radiol 2016;27:1518-30.

16. Ahmed O, Patel K, Rabei R, Patel MV, Ginsburg M, Clayton B, et al. Hemodialysis access maintenance in the medicare population: An analysis over a decade of trends by provider specialty and site of service. J Vasc Interv Radiol 2018;29:159-69.

17. Wallace JR, Chaer RA, Dillavou ED. Report on the hemodialysis reliable outflow (HeRO) experience in dialysis patients with central venous occlusions. J Vasc Surg 2013;58:742-7.

18. Katzman HE, McLafferty RB, Ross JR, Glickman MH,
Peden EK, Lawson JH, et al. Initial experience and outcome of a new hemodialysis access device for catheter-dependent patients. J Vasc Surg 2009;50:600-7, 607.e1.

19. Ladenheim ED, Lulic D, Lum C, Agrawal S. Primary and secondary patencies of transposed femoral vein fistulas are significantly greater than with the heRO graft. J Vasc Access 2017; 18:232-7.

20. Al Shakarchi J, Houston JG, Jones RG, Inston N. A review on the hemodialysis reliable outflow (HeRO) graft for haemodialysis vascular access. Eur J Vasc Endovasc Surg 2015;50:108-13.

21. Gibbens DT, Triolo J, Yu T, Depalma J, Iglasias J, Castner D, et al. Contemporary treatment of thrombosed hemodialysis grafts. Tech Vasc Interv Radiol 2001;4:122-6.

22. Coan KE, O'Donnell ME, Fankhauser GT, Bodnar Z, Chandrasekaran K, Stone WM, et al. Bilateral pulmonary emboli secondary to indwelling hemodialysis reliable outflow catheter. Vasc Endovascular Surg 2013;47:317-9.

23. Calderon K, Jhaveri KD, Mossey R. Pulmonary embolism following thrombolysis of dialysis access: Is anticoagulation really necessary? Semin Dial 2010;23:522-5.

24. Smits HF, Van Rijk PP, Van Isselt JW, Mali WP, Koomans HA, Blankestijn PJ, et al. Pulmonary embolism after thrombolysis of hemodialysis grafts. J Am Soc Nephrol 1997;8:1458-61.

25. Sadjadi SA, Sharif-Hassanabadi M. Fatal pulmonary embolism after hemodialysis vascular access declotting. Am J Case Rep 2014;15:172-5.

26. Gilmore B, Benrashid E, Youngwirth LM, Lawson JH. Paradoxical embolus following percutaneous thrombectomy of hemodialysis reliable outflow graft. J Vasc Access 2015;16:533-6.

27. Hsieh MY, Lai CL, Wu YW, Lin L, Ho MC, Wu CC, et al. Impact on pulmonary arterial pressures after repeated endovascular thrombectomy of dialysis grafts: A prospective follow-up study. J Vasc Interv Radiol 2014;25:1883-9.

28. Yigla M, Fruchter O, Aharonson D, Yanay N, Reisner SA, Lewin $\mathrm{M}$, et al. Pulmonary hypertension is an independent predictor of mortality in hemodialysis patients. Kidney Int 2009;75:969-75.

29. Harp RJ, Stavropoulos SW, Wasserstein AG, Clark TW. Pulmonary hypertension among end-stage renal failure patients following hemodialysis access thrombectomy. Cardiovasc Intervent Radiol 2005;28:17-22. 